\title{
Modeling Attacks on Physical Unclonable Functions
}

\author{
Ulrich Rührmair \\ Computer Science Departm. \\ TU München \\ 80333 München, Germany \\ ruehrmair@in.tum.de \\ Gideon Dror \\ The Academic College of \\ Tel-Aviv-Jaffa \\ Tel-Aviv 61083, Israel \\ gideon@mta.ac.il
}

\author{
Frank Sehnke \\ Computer Science Departm. \\ TU München \\ 80333 München, Germany \\ sehnke@in.tum.de \\ Srinivas Devadas \\ Department of EECS \\ MIT \\ Cambrige, MA, USA \\ devadas@mit.edu
}

\author{
Jan Sölter \\ Computer Science Departm. \\ TU München \\ 80333 München, Germany \\ jan_soelter@yahoo.com \\ Jürgen Schmidhuber \\ Computer Science Departm. \\ TU München \\ 80333 München, Germany \\ juergen@idsia.ch
}

\begin{abstract}
We show in this paper how several proposed Physical Unclonable Functions (PUFs) can be broken by numerical modeling attacks. Given a set of challenge-response pairs (CRPs) of a PUF, our attacks construct a computer algorithm which behaves indistinguishably from the original PUF on almost all CRPs. This algorithm can subsequently impersonate the PUF, and can be cloned and distributed arbitrarily. This breaks the security of essentially all applications and protocols that are based on the respective PUF.
\end{abstract}

The PUFs we attacked successfully include standard Arbiter PUFs and Ring Oscillator PUFs of arbitrary sizes, and XOR Arbiter PUFs, Lightweight Secure PUFs, and Feed-Forward Arbiter PUFs of up to a given size and complexity. Our attacks are based upon various machine learning techniques, including Logistic Regression and Evolution Strategies. Our work leads to new design requirements for secure electrical PUFs, and will be useful to PUF designers and attackers alike.

\section{Categories and Subject Descriptors}

C.3 [Special Purpose and Application-Based Systems]: Smartcards; B.7.m [Integrated Circuits]: Miscellaneous; E.3 [Data Encryption]: Code breaking

\section{General Terms}

Security, Theory, Design

\section{Keywords}

Physical Unclonable Functions, Machine Learning, Cryptanalysis, Physical Cryptography

\section{INTRODUCTION}

\subsection{Motivation and Background}

Electronic devices are now pervasive in our everyday life. They are an accessible target for adversaries, which raises a host of security and privacy issues. Classical cryptography offers several measures against these problems, but they all rest on the concept of a secret binary key: It is assumed that the devices can contain a piece of information that is, and remains, unknown to the adversary. Unfortunately, it can be difficult to uphold this requirement in practice. Physical attacks such as invasive, semi-invasive, or side-channel attacks, as well as software attacks like API-attacks and viruses, can lead to key exposure and full security breaks. The fact that the devices should be inexpensive, mobile, and cross-linked obviously aggravates the problem.

The described situation was one motivation that led to the development of Physical Unclonable Functions (PUFs). A PUF is a (partly) disordered physical system $S$ that can be challenged with so-called external stimuli or challenges $C_{i}$, upon which it reacts with corresponding responses termed $R_{C_{i}}$. Contrary to standard digital systems, a PUF's responses shall depend on the nanoscale structural disorder present in the PUF. This disorder cannot be cloned or reproduced exactly, not even by its original manufacturer, and is unique to each PUF. Assuming the stability of the PUF's responses, any PUF $S$ hence implements an individual function $F_{S}$ that maps challenges $C_{i}$ to responses $R_{C_{i}}$ of the PUF.

Due to its complex and disordered structure, a PUF can avoid some of the shortcomings associated with digital keys. For example, it is usually harder to read out, predict, or derive its responses than to obtain the values of digital keys stored in non-volatile memory. This fact has been exploited for various PUF-based security protocols. Prominent examples including schemes for identification and authentication $[1,2]$, key exchange or digital rights management purposes $[3]$.

\subsection{Strong PUFs, Controlled PUFs, and Weak PUFs}

There are several subtypes of PUFs, each with its own applications and security features. Three major types, which 
must explicitly be distinguished in this paper, are Strong PUFs [1, 2, 4] ${ }^{1}$, Controlled PUFs [3], and Weak PUFs [4], initially termed Physically Obfuscated Keys (POKs) [5].

\subsubsection{Strong PUFs}

Strong PUFs are disordered physical systems with a complex challenge-response behavior and very many possible challenges. Their security features are: (i) It must be impossible to physically clone a Strong PUF, i.e., to fabricate a second system which behaves indistinguishably from the original PUF in its challenge-response behavior. This restriction shall hold even for the original manufacturer of the PUF. (ii) A complete determination/measurement of all challengeresponse pairs (CRPs) within a limited time frame (such as several days or even weeks) must be impossible, even if one can challenge the PUF freely and has unrestricted access to its responses. This property is usually met by the large number of possible challenges and the finite read-out speed of a Strong PUF. (iii) It must be difficult to numerically predict the response $R_{C}$ of a Strong PUF to a randomly selected challenge $C$, even if many other CRPs are known.

Possible applications of Strong PUFs cover key establishment [1, 7], identification [1], and authentication [2]. They also include oblivious transfer [8] and any protocols derived from it, including zero-knowledge proofs, bit commitment, and secure multi-party computation [8]. In said applications, Strong PUFs can achieve secure protocols without the usual, standard computational assumptions concerning the factoring or discrete logarithm problem (albeit their security rests on other, independent computational and physical assumptions). Currently known electrical, circuit-based candidates for Strong PUFs are described in $[9,10,11,12,13]$.

\subsubsection{Controlled PUFs}

A Controlled PUF as described in [3] uses a Strong PUF as a building block, but adds control logic that surrounds the PUF. The logic prevents challenges from being applied freely to the PUF, and hinders direct read-out of its responses. This logic can be used to thwart modeling attacks. However, if the outputs of the embedded Strong PUF can be directly probed, then it may be possible to model the Strong PUF and break the Controlled PUF protocol.

\subsubsection{Weak PUFs}

Weak PUFs, finally, may have very few challenges - in the extreme case just one, fixed challenge. Their response(s) $R_{C_{i}}$ are used to derive a standard secret key, which is subsequently processed by the embedding system in the usual fashion, e.g., as a secret input for some cryptoscheme. Contrary to Strong PUFs, the responses of a Weak PUF are never meant to be given directly to the outside world.

Weak PUFs essentially are a special form of non-volatile key storage. Their advantage is that they may be harder to read out invasively than non-volatile memory like EEPROM. Typical examples include the SRAM PUF [14, 4], Butterfly PUF [15] and Coating PUF [16]. Integrated Strong PUFs have been suggested to build Weak PUFs or Physically Ob-

${ }^{1}$ Strong PUFs have also been referred to as Physical Random Functions [5], or Physical One-Way Functions [6]. fuscated Keys (POKs), in which case only a small subset of all possible challenges is used [5, 9].

One important aspect of Weak PUFs is error correction and stability. Since their responses are processed internally as a secret key, error correction must be carried out on-chip and with perfect precision. This often requires the storage of error-correcting helper data in non-volatile memory on the chip. Strong PUFs usually allow error correction schemes that are carried out by the external recipients of their responses.

\subsection{Modeling Attacks on PUFs}

Modeling attacks on PUFs presume that an adversary Eve has, in one way or the other, collected a subset of all CRPs of the PUF, and tries to derive a numerical model from this data, i.e., a computer algorithm which correctly predicts the PUF's responses to arbitrary challenges with high probability. If successful, this breaks the security of the PUF and of any protocols built on it. It is known from earlier work that machine learning (ML) techniques are a natural and powerful tool for such modeling attacks $[5,17,18,19,20]$. How the required CRPs can be collected depends on the type of PUF under attack.

Strong PUFs. Strong PUFs usually have no protection mechanisms that restricts Eve in challenging them or in reading out their responses. Their responses are freely accessible from the outside, and are usually not post-processed on chip $[1,9,10,11,12,13]$. Most electrical Strong PUFs further operate at frequencies of a few $\mathrm{MHz}$ [12]. Therefore even short physical access periods enable the read-out of many CRPs. Another potential CRP source is simple protocol eavesdropping, for example on standard Strong PUF-based identification protocols, where the CRPs are sent in the clear [1]. Eavesdropping on responses, as well as physical access to the PUF that allows the adversary to apply arbitrary challenges and read out their responses, is part of the established attack model for Strong PUFs.

Controlled PUFs. For any adversary that is restricted to non-invasive CRP measurement, modeling attacks can be successfully disabled if one uses a secure one-way hash over the outputs of the PUF to create a Controlled PUF. We note that this requires error correction of the PUF outputs which are inherently noisy [3]. Successful application of our techniques to a Controlled PUF only becomes possible if Eve can probe the internal, digital response signals of the underlying Strong PUF on their way to the control logic. Even though this is a significant assumption, probing digital signals is still easier than measuring continuous analog parameters within the underlying Strong PUF, for example determining its delay values. Physical access to the PUF is part of the natural attack model on PUFs, as mentioned above.

Weak PUFs. Weak PUFs are only susceptible to model building attacks if a Strong PUF, embedded in some hardware system, is used to derive the physically obfuscated key. This method has been suggested in [5,9]. In this case, the 
internal digital response signals of the Strong PUF to injected challenges have to be probed.

We stress that purely numerical modeling attacks, as presented in this paper, are not relevant for Weak PUFs with just one challenge (such as the Coating PUF, SRAM PUF, or Butterfly PUF). This does not necessarily imply that these PUFs are more secure than Strong PUFs or Controlled PUFs, however. Other attack strategies can be applied, including invasive, side-channel and virus attacks, but they are not the topic of this paper. For example, probing the output of the SRAM cell prior to storing the value in a register can break the security of the cryptographic protocol that uses these outputs as a key. We also note that attacking a Controlled PUF via modeling attacks that target the underlying Strong PUF requires substantially more signal probing than breaking a Weak PUF that possesses just one challenge.

\subsection{Our Contributions and Related Work}

We describe successful modeling attacks on several known electrical candidates for Strong PUFs, including Arbiter PUFs, XOR Arbiter PUFs, Feed-Forward Arbiter PUFs, Lightweight Secure PUFs, and Ring Oscillator PUFs. Our attacks work for PUFs of up to a given number of inputs (or stages) or complexity. The prediction rates of our machine learned models significantly exceed the known or derived stability of the respective PUFs in silicon in these ranges.

Our attacks are very feasible on the CRP side. They require an amount of CRPs that grows only linearly or log-linearly in the relevant structural parameters of the attacked PUFs, such as their numbers of stages, XORs, feed-forward loops, or ring oscillators. The computation times needed to derive the models (i.e., to train the employed ML algorithms) are low-degree polynomial, with one exception: The computation times for attacking XOR Arbiter and Lightweight Secure PUFs grow, in approximation for medium number of XORs and large number of stages, super-polynomial in the number of XORs. But the instability of these PUFs also increases exponentially in their number of XORs, whence this parameter cannot be raised at will in practical applications. Still, it turns out that the number of stages in these two types of PUFs can be increased without significant effect on their instability, providing a potential lever for making these PUFs more secure without destroying their practicality. Our work thus also points to design requirements by which the security of XOR Arbiter PUFs and Lightweight Secure PUFs against modeling attacks could be upheld in the near future.

Our results break the security of any Strong PUF-type protocol that is based on one of the broken PUFs. This includes any identification, authentication, key exchange or digital rights management protocols, such as the ones described in $[1,2,6,7,11]$. Under the assumptions and attack scenarios described in Section 1.3, our findings also restrict the use of the broken Strong PUF architectures within Controlled PUFs and as Weak PUFs, if we assume that digital values can be probed.

Related Work on Modeling Attacks. Earlier work on PUF modeling attacks, such as $[11,17,18,19]$, described success- ful attacks on standard Arbiter PUFs and on Feed-Forward Arbiter PUFs with one loop. But these approaches did not generalize to Feed-Forward Arbiter PUFs with more than two loops. The XOR Arbiter PUF, Lightweight PUF, FeedForward Arbiter PUF with more than two Feed-Forward Loops, and Ring Oscillator PUF have not been cryptanalyzed thus far. No scalability analyses of the required CRPs and computation times had been performed in previous works.

Entropy Analysis vs. Modeling Attacks. Another useful approach to evaluate PUF security is entropy analysis. Two variants exist: First, to analyze the internal entropy of the PUF. This is similar to the established physical entropy analysis in solid-state systems. A second option is to analyze the statistical entropy of all challenge-response pairs of a PUF; how many of them are independent?

Entropy analysis is a valuable tool for PUF analysis, but it differs from our approach in two aspects. First, it is nonconstructive in the sense that it does not tell you how to break a PUF, even if the entropy score is low. Modeling attacks, to the contrary, actually break PUFs. Second, it is not clear if the internal entropy of a circuit-based Strong PUF is a good estimate for its security. Equivalently, is the entropy of an AES secret key a good estimate of the AES security? The security of a Strong PUF comes from an interplay between its random internal parameters (which can be viewed as its entropy), and its internal model or internal functionality. It is not the internal entropy alone that determines the security. As an example, compare an 8XOR, 256-bit XOR PUF to a standard PUF with bitlength of $8 \cdot 256=2048$. Both have the same internal entropy, but very different security properties, as we show in the sequel.

\subsection{Organization of the Paper}

The paper is organized as follows. We describe the methodology of our ML experiments in Section 2. In Sections 3 to 7, we present our results for various Strong PUF candidates. They deal with Arbiter PUFs, XOR Arbiter PUFs, Lightweight Arbiter PUFs, Feed-Forward Arbiter PUFs and Ring Oscillator PUFs, in sequence. We conclude with a summary and discussion of our results in Section 8.

\section{METHODOLOGY SECTION}

\subsection{Employed Machine Learning Methods}

\subsubsection{Logistic Regression}

Logistic Regression (LR) is a well-investigated supervised machine learning framework, which has been described, for example, in [21]. In its application to PUFs with single-bit outputs, each challenge $C=b_{1} \cdots b_{k}$ is assigned a probability $p(C, t \mid \vec{w})$ that it generates a output $t \in\{-1,1\}$ (for technical reasons, one makes the convention that $t \in\{-1,1\}$ instead of $\{0,1\})$. The vector $\vec{w}$ thereby encodes the relevant internal parameters, for example the particular runtime delays, of the individual PUF. The probability is given by the logistic sigmoid acting on a function $f(\vec{w})$ parametrized by the vector $\vec{w}$ as $p(C, t \mid \vec{w})=\sigma(t f)=\left(1+e^{-t f}\right)^{-1}$. Thereby $f$ determines through $f=0$ a decision boundary of equal output probabilities. For a given training set $\mathcal{M}$ of CRPs the boundary is positioned by choosing the parameter vector 
$\vec{w}$ in such a way that the likelihood of observing this set is maximal, respectively the negative log-likelihood is minimal:

$$
\hat{\vec{w}}=\operatorname{argmin}_{\vec{w}} l(\mathcal{M}, \vec{w})=\operatorname{argmin}_{\vec{w}} \sum_{(C, t) \in \mathcal{M}}-\ln (\sigma(t f(\vec{w}, C)))
$$

As there is no analytical solution to determine the optimal parameter vector $\hat{\vec{w}}$, it has to be optimized iteratively, e.g., using the gradient information

$$
\nabla l(\mathcal{M}, \vec{w})=\sum_{(C, t) \in \mathcal{M}} t(\sigma(t f(\vec{w}, C))-1) \nabla f(\vec{w}, C)
$$

From the different optimization methods which we tested in our ML experiments (standard gradient descent, iterative reweighted least squares, RProp [21] [22]), RProp gradient descent performed best. Logistic regression has the asset that the examined problems need not be (approximately) linearly separable in feature space, as is required for successful application of SVMs, but merely differentiable.

In our ML experiments, we used an implementation of LR with RProp programmed in our group, which has been put online, see [23]. The iteration is continued until we reach a point of convergence, i.e., until the averaged prediction rate of two consecutive blocks of five consecutive iterations does not increase anymore for the first time. If the reached performance after convergence on the training set is not sufficient, the process is started anew. After convergence to a good solution on the training set, the prediction error is evaluated on the test set.

The whole process is similar to training an Artificial Neural Network (ANN) [21]. The model of the PUF resembles the network with the runtime delays resembling the weights of an ANN. Similar to ANNs, we found that RProp makes a very big difference in convergence speed and stability of the LR (several XOR-PUFs were only learnable with RProp). But even with RProp the delay set can end up in a region of the search space where no helpful gradient information is available (local minimum). In such a case we encounter the above described situation of converging on a not sufficiently accurate solution and have to restart the process.

\subsubsection{Evolution Strategies}

Evolution Strategies (ES) [24, 25] belong to an ML subfield known as population-based heuristics. They are inspired by the evolutionary adaptation of a population of individuals to certain environmental conditions. In our case, one individual in the population is given by a concrete instantiation of the runtime delays in a PUF, i.e., by a concrete instantiation of the vector $\vec{w}$ appearing in Eqns. 1 and 2. The environmental fitness of the individual is determined by how well it (re-)produces the correct CRPs of the target PUF on a fixed training set of CRPs. ES runs through several evolutionary cycles or so-called generations. With a growing number of generations, the challenge-response behavior of the best individuals in the population better and better approximates the target PUF. ES is a randomized method that neither requires an (approximately) linearly separable problem (like Support Vector Machines), nor a differentiable model (such as LR with gradient descent); a merely parameterizable model suffices. Since all known electrical PUFs are easily parameterizable, ES is a very well-suited attack method.

We employed an in-house implementation of ES that is available from our machine learning library PyBrain [26]. The meta-parameters in all applications of ES throughout this paper are $(6,36)$-selection and a global mutation operator with $\tau=\frac{1}{\sqrt{n}}$. We furthermore used a technique called Lazy Evaluation (LE). LE means that not all CRPs of the training set are used to evaluate an individual's environmental fitness; instead, only a randomly chosen subset is used for evaluation, that changes in every generation. In this paper, we always used subsets of size 2,000 CRPs, and indicated this also in the caption of the respective tables.

\subsection{Employed Computational Resources}

We used two hardware systems to carry out our experiments: A stand-alone, consumer INTEL Quadcore Q9300 worth less than 1,000 Euros. Experiments run on this system are marked with the term "HW $\star$ ". Secondly, a 30node cluster of AMD Opteron Quadcores, which represents a worth of around 30,000 Euros. Results that were obtained by this hardware are indicated by the term "HW ם". All computation times are calculated for one core of one processor of the corresponding hardware.

\subsection{PUF Descriptions and Models}

Arbiter PUFs. Arbiter PUFs (Arb-PUFs) were first introduced in [11] [12] [9]. They consist of a sequence of $k$ stages, for example multiplexers. Two electrical signals race simultaneously and in parallel through these stages. Their exact paths are determined by a sequence of $k$ external bits $b_{1} \cdots b_{k}$ applied to the stages, whereby the $i$-th bit is applied at the $i$-th stage. After the last stage, an "arbiter element" consisting of a latch determines whether the upper or lower signal arrived first and correspondingly outputs a zero or a one. The external bits are usually regarded as the challenge $C$ of this PUF, i.e., $C=b_{1} \cdots b_{k}$, and the output of the arbiter element is interpreted as their response $R$. See [11] [12] [9] for details.

It has become standard to describe the functionality of ArbPUFs via an additive linear delay model [17] [10] [19]. The overall delays of the signals are modeled as the sum of the delays in the stages. In this model, one can express the final delay difference $\Delta$ between the upper and the lower path in a $k$-bit Arb-PUF as $\Delta=\vec{w}^{T} \vec{\Phi}$, where $\vec{w}$ and $\vec{\Phi}$ are of dimension $k+1$. The parameter vector $\vec{w}$ encodes the delays for the subcomponents in the Arb-PUF stages, whereas the feature vector $\vec{\Phi}$ is solely a function of the applied $k$-bit challenge $C$ [17] [10] [19].

In greater detail, the following holds. We denote by $\delta_{i}^{0 / 1}$ the runtime delay in stage $i$ for the crossed (1) respectively uncrossed (0) signal path. Then

$$
\vec{w}=\left(w^{1}, w^{2}, \ldots, w^{k}, w^{k+1}\right)^{T},
$$

where $w^{1}=\frac{\delta_{1}^{0}-\delta_{1}^{1}}{2}, w^{i}=\frac{\delta_{i-1}^{0}+\delta_{i-1}^{1}+\delta_{i}^{0}-\delta_{i}^{1}}{2}$ for all $i=$ 
$2, \ldots, k$, and $w^{k+1}=\frac{\delta_{k}^{0}+\delta_{k}^{1}}{2}$. Furthermore,

$$
\vec{\Phi}(\vec{C})=\left(\Phi^{1}(\vec{C}), \ldots, \Phi^{k}(\vec{C}), 1\right)^{T},
$$

where $\Phi^{l}(\vec{C})=\prod_{i=l}^{k}\left(1-2 b_{i}\right)$ for $l=1, \ldots, k$.

The output $t$ of an Arb-PUF is determined by the sign of the final delay difference $\Delta$. We make the technical convention of saying that $t=-1$ when the Arb-PUF output is actually 0 , and $t=1$ when the Arb-PUF output is 1 :

$$
t=\operatorname{sgn}(\Delta)=\operatorname{sgn}\left(\vec{w}^{T} \vec{\Phi}\right) .
$$

Eqn. 5 shows that the vector $\vec{w}$ via $\vec{w}^{T} \vec{\Phi}=0$ determines a separating hyperplane in the space of all feature vectors $\vec{\Phi}$. Any challenges $C$ that have their feature vector located on the one side of that plane give response $t=-1$, those with feature vectors on the other side $t=1$. Determination of this hyperplane allows prediction of the PUF.

XOR Arbiter PUFs. One possibility to strengthen the resilience of arbiter architectures against machine learning, which has been suggested in [9], is to employ $l$ individual Arb-PUFs in parallel, each with $k$ stages. The same challenge $C$ is applied to all of them, and their individual outputs $t_{i}$ are XORed in order to produce a global response $t_{X O R}$. We denote such an architecture as $l$-XOR Arb-PUF

A formal model for the XOR Arb-PUF can be derived as follows. Making the convention $t_{i} \in\{-1,1\}$ as done earlier, it holds that $t_{X O R}=\prod_{i=1}^{l} t_{i}$. This leads with equation (5) to a parametric model of an $l$-XOR Arb-PUF, where $\vec{w}_{i}$ and $\vec{\Phi}_{i}$ denote the parameter and feature vector, respectively, for the $i$-th Arb PUF:

$$
\begin{aligned}
t_{X O R} & =\prod_{i=1}^{l} \operatorname{sgn}\left(\vec{w}_{i}^{T} \vec{\Phi}_{i}\right)=\operatorname{sgn}\left(\prod_{i=1}^{l} \vec{w}_{i}^{T} \vec{\Phi}_{i}\right) \\
& =\operatorname{sgn}(\underbrace{\bigotimes_{i=1}^{l} \vec{w}_{i}^{T}}_{\vec{w}_{X O R}} \underbrace{\bigotimes_{i=1}^{l} \vec{\Phi}_{i}}_{\vec{\Phi}_{X O R}})=\operatorname{sgn}\left(\vec{w}_{X O R}^{T} \vec{\Phi}_{X O R}\right)(7)
\end{aligned}
$$

Whereas (6) gives a non-linear decision boundary with $l(k+$ 1) parameters, (7) defines a linear decision boundary by a separating hyperplane $\vec{w}_{X O R}$ which is of dimension $(k+1)^{l}$.

Lightweight Secure PUFs. Another type of PUF, which we term Lightweight Secure PUF or Lightweight PUF for short, has been introduced in [10]. It is similar to the XOR Arb-PUF of the last paragraph. At its heart are $l$ individual standard Arb-PUFs arranged in parallel, each with $k$ stages, which produce $l$ individual outputs $r_{1}, \ldots, r_{l}$. These individual outputs are XORed to produce a multi-bit response $o_{1}, \ldots, o_{m}$ of the Lightweight PUF, according to the formula

$$
o_{j}=\bigoplus_{i=1, \ldots, x} r_{(j+s+i) \bmod l} \quad \text { for } j=1, \ldots, m .
$$

Thereby the values for $m$ (the number of output bits of the Lightweight PUF), $x$ (the number of values $r_{j}$ that influence each single output bit) and $s$ (the circular shift in choosing the $x$ values $r_{j}$ ) are variable design parameters.
Another difference to the XOR Arb-PUFs lies in the $l$ inputs $C_{1}=b_{1}^{1} \cdots b_{k}^{1}, C_{2}=b_{1}^{2} \cdots b_{k}^{2}, \ldots, C_{l}=b_{1}^{l} \cdots b_{k}^{l}$ which are applied to the $l$ individual Arb-PUFs. Contrary to XOR Arb-PUFs, it does not hold that $C_{1}=C_{2}=\ldots=C_{l}=C$, but a more complicated input mapping that derives the individual inputs $C_{i}$ from the global input $C$ is applied. This input mapping constitutes the most significant difference between the Lightweight PUF and the XOR Arb PUF. We refer the reader to [10] for further details.

In order to predict the whole output of the Lightweight PUF, one can apply similar models and ML techniques as in the last section to predict its single output bits $o_{j}$. While the probability to predict the full output of course decreases exponentially in the misclassification rate of a single bit, the stability of the full output of the Lightweight PUF also decreases exponentially in the same parameters. It therefore seems fair to attack it in the described manner; in any case, our results challenge the bit security of the Lightweight PUF.

Feed Forward Arbiter PUFs. Feed Forward Arbiter PUFs (FF Arb-PUFs) were introduced in [11] [12] [17] and further discussed in [19]. Some of their multiplexers are not switched in dependence of an external challenge bit, but as a function of the delay differences accumulated in earlier parts of the circuit. Additional arbiter components evaluate these delay differences, and their output bit is fed into said multiplexers in a "feed-forward loop" (FF-loop). We note that an FF Arb-PUF with $k$-bit challenges $C=b_{1} \cdots b_{k}$ and $l$ loops has $s=k+l$ multiplexers or stages.

The described dependency makes natural architecture models of FF Arb-PUFs no longer differentiable. Consequently, FF Arb-PUFs cannot be attacked generically with ML methods that require linearly separable or differentiable models (like SVMs or LR), even though such models can be found in special cases, for example for small numbers of non-overlapping loops.

The number of loops as well as the starting and end point of the FF-loops are variable design parameters, and a host of different architectures for an FF Arb-PUF with a moderate or even large number of loops are possible. The architecture we investigated in this paper consists of loops that are distributed at equal distances over the structure, and which just overlap each other: If the starting point of loop $m$ lies in between stages $n$ and $n+1$, then the previous loop $m-1$ has its end point in the immediately following stage $n+1$. This seemed the natural and straightforward architectural choice; future experiments will determine whether this is indeed the optimal (i.e., most secure) architecture.

Ring Oscillator PUFs. Ring Oscillator PUFs (RO-PUFs) were discussed in [9]. They are based on the influence of fabrication variations on the frequency of several, identically designed ring oscillators. While [9] describes the use of Ring Oscillator PUFs in the context of Controlled PUFs and limited-count authentication, it is worth analyzing them as candidate Strong PUFs. A RO-PUF consists of $k$ oscillators, each of which has its own, unique frequency caused by manufacturing variations. The input of a RO-PUF consists 
of a tuple $(i, j)$, which selects two of the $k$ oscillators. Their frequencies are compared, and the output of the RO-PUF is " 0 " if the former oscillates faster than the latter, and "1" else. A ring oscillator can be modeled in a straightforward fashion by a tuple of frequencies $\left(f_{1}, \ldots, f_{k}\right)$. Its output on input $(i, j)$ is " 0 " if $f_{i}>f_{j}$, and " 1 " else.

\subsection{CRP Generation, Prediction Error, and Number of CRPs}

Given a PUF-architecture that should be examined, the challenge-response pairs (CRPs) that we used in our ML experiments were generated in the following fashion: (i) The delay values for this PUF architecture were chosen pseudorandomly according to a standard normal distribution. We sometimes refer to this as choosing a certain PUF instance in the paper. In the language of Equ. 3, it amounts to choosing the entries $w^{i}$ pseudo-randomly. (ii) If a response of this PUF instance to a given challenge is needed, it is calculated by use of the delays selected in step (i), and by application of a linear additive delay model [13]: The delays of the two electrical signal paths are simply added up and compared.

We use the following definitions throughout the paper: The prediction error $\epsilon$ is the ratio of incorrect responses of the trained ML algorithm when evaluated on the test set. For all applications of LR, the test set each time consisted of 10,000 randomly chosen CRPs. For all applications of ES (i.e., for the Feed-Forward Arbiter PUF), the test set each time consisted of 8,000 randomly chosen CRPs. The prediction rate is $1-\epsilon$.

$N_{C R P}$ (or simply "CRPs") denotes the number of CRPs employed by the attacker in his respective attack, for example in order to achieve a certain prediction rate. This nomenclature holds throughout the whole paper. Nevertheless, one subtle difference should be made explicit: In all applications of LR (i.e., in Sections 3 to 5 ), $N_{C R P}$ is equal to the size of the training set of the ML algorithm, as one would usually expect. In the applications of ES (i.e., in Section 6), however, the situation is more involved. The attacker needs a test set himself in order to determine which of his many random runs was the best. The value $N_{C R P}$ given in the tables and formulas of Section 6 hence reflects the sum of the sizes of the training set and the test set employed by the attacker.

\section{ARBITER PUFS}

\subsection{Machine Learning Results}

To determine the separating hyperplane $\vec{w}^{T} \vec{\Phi}=0$, we applied SVMs, LR and ES. LR achieved the best results, which are shown in Table 1 . We chose three different prediction rates as targets: $95 \%$ is roughly the environmental stability of a 64-bit Arbiter PUF when exposed to a temperature variation of $45 \mathrm{C}$ and voltage variation of $\pm 2 \%^{2}$. The values $99 \%$ and $99.9 \%$, respectively, represent benchmarks for optimized ML results. All figures in Table 1 were obtained by averaging over 5 different training sets. Accuracies were estimated using test sets of 10,000 CRPs.

\footnotetext{
${ }^{2}$ The exact figures reported in [17] are: $4.57 \% \mathrm{CRP}$ variation for a temperature variation of $45 \mathrm{C}$, and $2.16 \%$ for a voltage variation of $\pm 2 \%$.
}

\begin{tabular}{|c|c|c|c|c|}
\hline $\begin{array}{c}\text { ML } \\
\text { Method }\end{array}$ & $\begin{array}{c}\text { No. of } \\
\text { Stages }\end{array}$ & $\begin{array}{c}\text { Prediction } \\
\text { Rate }\end{array}$ & CRPs & $\begin{array}{c}\text { Training } \\
\text { Time }\end{array}$ \\
\hline \multirow{2}{*}{ LR } & \multirow{2}{*}{64} & $95 \%$ & 640 & $0.01 \mathrm{sec}$ \\
& & $99 \%$ & 2,555 & $0.13 \mathrm{sec}$ \\
& & $99.9 \%$ & 18,050 & $0.60 \mathrm{sec}$ \\
\hline \multirow{2}{*}{ LR } & \multirow{2}{*}{128} & $95 \%$ & 1,350 & $0.06 \mathrm{sec}$ \\
& & $99.9 \%$ & 5,570 & $0.51 \mathrm{sec}$ \\
& & \multicolumn{3}{|c}{} \\
\hline
\end{tabular}

Table 1: LR on Arb PUFs with 64 and 128 stages. We used HW $\star$.

\subsection{Scalability}

We also executed scalability experiments with LR, which are displayed in Fig. 1 and Fig. 2. They show that the relevant parameters - the required number of CRPs in the training set and the computational complexity, i.e., the number of basic operations - grow both linearly or low-degree polynomially in the misclassification rate $\epsilon$ and the length $k$ of the Arb PUF. Theoretical considerations (dimension of the feature space, Vapnik-Chervonenkis dimension) suggest that the minimal number of CRPs $N_{C R P}$ that is necessary to model a $k$-stage arbiter with a misclassification rate of $\epsilon$ should obey the relation

$$
N_{C R P}=O(k / \epsilon) .
$$

This was confirmed by our experimental results.

In practical PUF applications, it is essential to know the concrete number of CRPs that may become known before the PUF-security breaks down. Assuming an approximate linear functional dependency $y=a x+c$ in the double logarithmic plot of Fig. 1 with a slope of $a=-1$, we obtained the following empirical formula (10). It gives the approximate number of CRPs $N_{C R P}$ that is required to learn a $k$-stage arbiter PUF with error rate $\epsilon$ :

$$
N_{C R P} \approx 0.5 \cdot \frac{k+1}{\epsilon}
$$

Our experiments also showed that the training time of the ML algorithms, measured in the number of basic operations

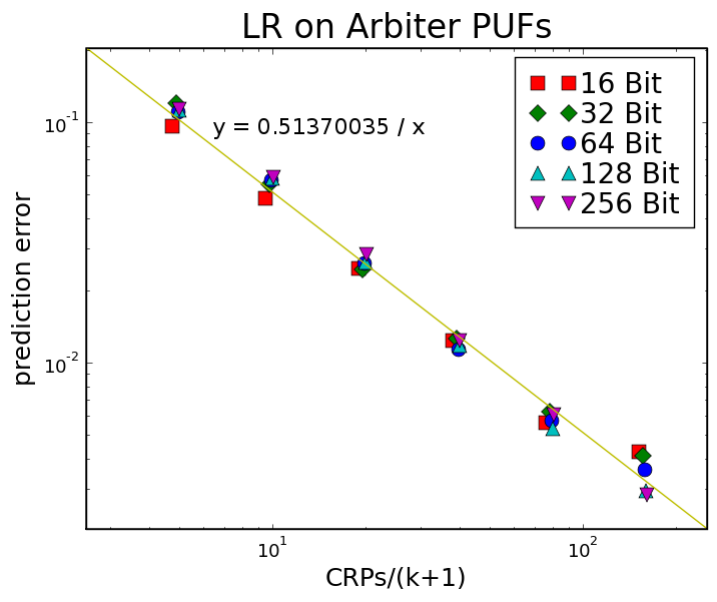

Figure 1: Double logarithmic plot of misclassification rate $\epsilon$ on the ratio of training CRPs $N_{C R P}$ and $\operatorname{dim}(\Phi)=k+1$. 


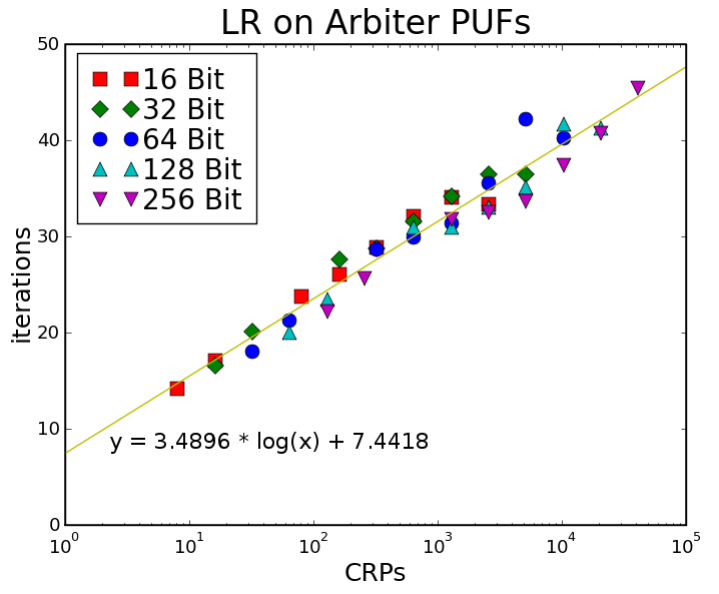

Figure 2: No. of iterations of the LR algorithm until "convergence" occurs (see section 2), plotted in dependence of the training set size $N_{C R P}$.

$N_{B O P}$, grows slowly. It is determined by the following two factors: (i) The evaluation of the current model's likelihood (Eqn. 1) and its gradient (Eqn. 2), and (ii) the number of iterations of the optimization procedure before convergence occurs (see section 2.1.1). The former is both a sum over a function of the feature vectors $\vec{\Phi}$ for all $N_{C R P}$, and therefore has complexity $O\left(k \cdot N_{C R P}\right)$. On the basis of the data shown in Figure 2, we may further estimate that the numbers of iterations increases proportional to the logarithm of the number of CRPs $N_{C R P}$. Together, this yields an overall complexity of

$$
N_{B O P}=O\left(\frac{k^{2}}{\epsilon} \cdot \log \frac{k}{\epsilon}\right) .
$$

\section{XOR ARBITER PUFS}

\subsection{Machine Learning Results}

In the application of SVMs and ES to XOR Arb-PUFs, we were able to break small instances, for example XOR ArbPUFs with 2 or 3 XORs and 64 stages. LR significantly outperformed the other two methods. The key observation is that instead of determining the linear decision boundary (Eqn. 7), one can also specify the non-linear boundary (Eqn. 6). This is done by setting the LR decision boundary $f=$ $\prod_{i=1}^{l} \vec{w}_{i}^{T} \vec{\Phi}_{i}$. The results are displayed in Table 2 .

\subsection{Performance on Error-Inflicted CRPs}

\begin{tabular}{|c|c|c|c|c|c|}
\hline $\begin{array}{c}\text { ML } \\
\text { Method }\end{array}$ & $\begin{array}{l}\text { No. of } \\
\text { Stages }\end{array}$ & $\begin{array}{l}\text { Pred. } \\
\text { Rate }\end{array}$ & $\begin{array}{l}\text { No. of } \\
\text { XORs }\end{array}$ & CRPs & $\begin{array}{c}\text { Training } \\
\text { Time }\end{array}$ \\
\hline \multirow{3}{*}{ LR } & \multirow{3}{*}{64} & \multirow{3}{*}{$99 \%$} & 4 & 12,000 & $3: 42 \mathrm{~min}$ \\
\hline & & & 5 & 80,000 & 2:08 hrs \\
\hline & & & 6 & 200,000 & 31:01 hrs \\
\hline \multirow{3}{*}{$\mathrm{LR}$} & \multirow{3}{*}{128} & \multirow{3}{*}{$99 \%$} & 4 & 24,000 & $2: 52 \mathrm{hrs}$ \\
\hline & & & 5 & 500,000 & $16: 36 \mathrm{hrs}$ \\
\hline & & & 6 & - & - \\
\hline
\end{tabular}

Table 2: LR on XOR Arbiter PUFs. Training times are averaged over different PUF-instances. HW $\star$.

\begin{tabular}{|c|c|c|c|c|c|}
\hline CRPs & & \multicolumn{3}{|c|}{ Percentage of error-inflicted CRPs } \\
\cline { 3 - 6 }$\left(\times 10^{3}\right)$ & & $0 \%$ & $2 \%$ & $5 \%$ & $10 \%$ \\
\hline \multirow{5}{*}{24} & Best Pred. & $98.76 \%$ & $92.83 \%$ & $88.05 \%$ & - \\
& Aver. Pred. & $98.62 \%$ & $91.37 \%$ & $88.05 \%$ & - \\
& Succ. Trials & $0.6 \%$ & $0.8 \%$ & $0.2 \%$ & $0.0 \%$ \\
& Instances & $40.0 \%$ & $25.0 \%$ & $5.0 \%$ & $0.0 \%$ \\
\hline \multirow{5}{*}{50} & Best Pred. & $99.49 \%$ & $95.17 \%$ & $92.67 \%$ & $89.89 \%$ \\
& Aver. Pred. & $99.37 \%$ & $94.39 \%$ & $91.62 \%$ & $88.20 \%$ \\
& Succ. Trials & $12.4 \%$ & $13.9 \%$ & $10.0 \%$ & $4.6 \%$ \\
& Instances & $100.0 \%$ & $62.5 \%$ & $50.0 \%$ & $20.0 \%$ \\
\hline \multirow{5}{*}{200} & Best Pred. & $99.88 \%$ & $97.74 \%$ & $96.01 \%$ & $94.61 \%$ \\
& Aver. Pred. & $99.78 \%$ & $97.34 \%$ & $95.69 \%$ & $93.75 \%$ \\
& Succ. Trials & $100.0 \%$ & $87.0 \%$ & $87.0 \%$ & $71.4 \%$ \\
& Instances & $100.0 \%$ & $100.0 \%$ & $100.0 \%$ & $100.0 \%$ \\
\hline
\end{tabular}

Table 3: LR on 128-bit, 4-XOR Arb PUFs with different levels of error in the training set. We show the best and average prediction rates of 40 randomly chosen instances, the percentage of successful trials over all instances, and the percentage of and instances that converged to a sufficient optimum in at least one trial. We used HW $\square$.

\begin{tabular}{|c|c|c|c|c|c|}
\hline CRPs & & \multicolumn{4}{|c|}{ Percentage of error-inflicted CRPs } \\
\cline { 3 - 6 }$\left(\mathrm{x} 10^{3}\right)$ & & $0 \%$ & $2 \%$ & $5 \%$ & $10 \%$ \\
\hline \multirow{5}{*}{500} & Best Pred. & $99.90 \%$ & $97.55 \%$ & $96.48 \%$ & $93.12 \%$ \\
& Aver. Pred. & $99.84 \%$ & $97.33 \%$ & $95.84 \%$ & $93.12 \%$ \\
& Succ. Trials & $7.0 \%$ & $2.9 \%$ & $0.9 \%$ & $0.7 \%$ \\
& Instances & $20.0 \%$ & $20.0 \%$ & $10.0 \%$ & $5.0 \%$ \\
\hline
\end{tabular}

Table 4: LR on 128-bit, 5-XOR Arb PUFs with different amounts of error in the training set. Rest as in the caption of Table 3 . We used HW $\square$.

The CRPs used in Section 4.1 have been generated pseudorandomly via an additive, linear delay model of the PUF. This deviates from reality in two aspects: First of all, the CRPs obtained from real PUFs are subject to noise and random errors. Secondly, the linear model matches the phenomena on a real circuit very closely [17], but not perfectly. This leads to a deviation of any real system from the linear model on a small percentage of all CRPs.

In order to mimic this situation, we investigated the ML performance when a small error is injected artificially into the training sets. A given percentage of responses in the training set were chosen randomly, and their bit values were flipped. Afterwards, the ML performance on the unaltered, error-free test sets was evaluated. The results are displayed in Tables 3 and 4 . They show that LR can cope very well with errors, provided that around 3 to 4 times more CRPs are used. The required convergence times on error inflicted training sets did not change substantially compared to error free training sets of the same sizes.

\subsection{Scalability}

Figures 4 and 5 display the results of our scaling experiments with LR. Again, the smallest number of CRPs in the training set $N_{C R P}$ needed to achieve predictions with a misclassification rate $\epsilon$ scales linearly with the number of parameters of the problem (the product of the number of 


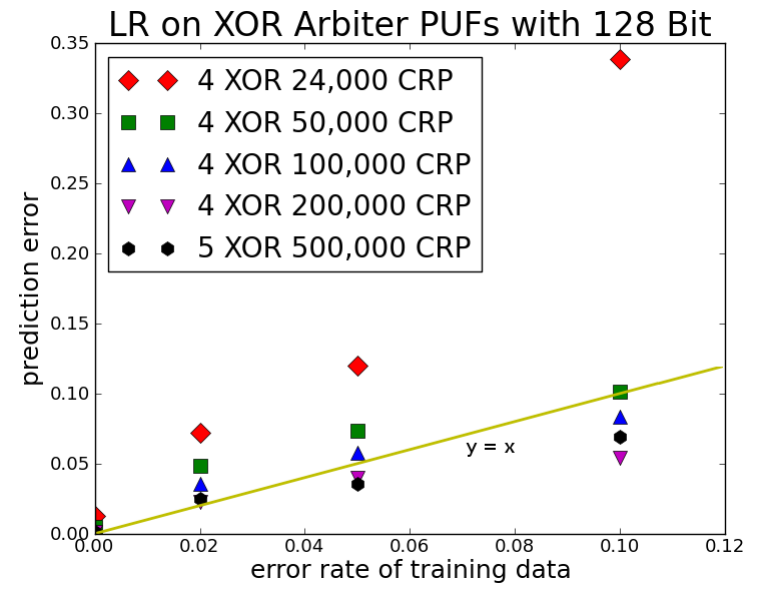

Figure 3: Graphical illustration of the effect of error on LR in the training set, with chosen data points from Tables 3 and 4 . We used HW $\square$.

stages $k$ and the number of XORed Arb-PUFs $l$ ):

$$
N_{C R P} \sim \frac{(k+1) \cdot l}{\epsilon} .
$$

But, in contrast to standard Arb-PUFs, optimizing the nonlinear decision boundary (6) on the training set now is a non-convex problem, so that the LR algorithm is not guaranteed to find (an attractor of) the global optimum in its first trial. It needs to be iteratively restarted $N_{\text {trial }}$ times. $N_{\text {trial }}$ thereby can be expected to not only depend on $k$ and $l$, but also on the size $N_{C R P}$ of the employed training set.

As it is argued in greater detail in [20], the success rate $\left(=1 / N_{\text {trial }}\right)$ of finding (an attractor of) the global optimum seems indeed determined by the ratio of dimensions gradient information $\left(\propto N_{C R P}\right.$ as the gradient is a linear combination of the feature vector) and the dimension $d_{\Phi}$ in which the problem is linear separable. The dimension $d_{\Phi}$ is the

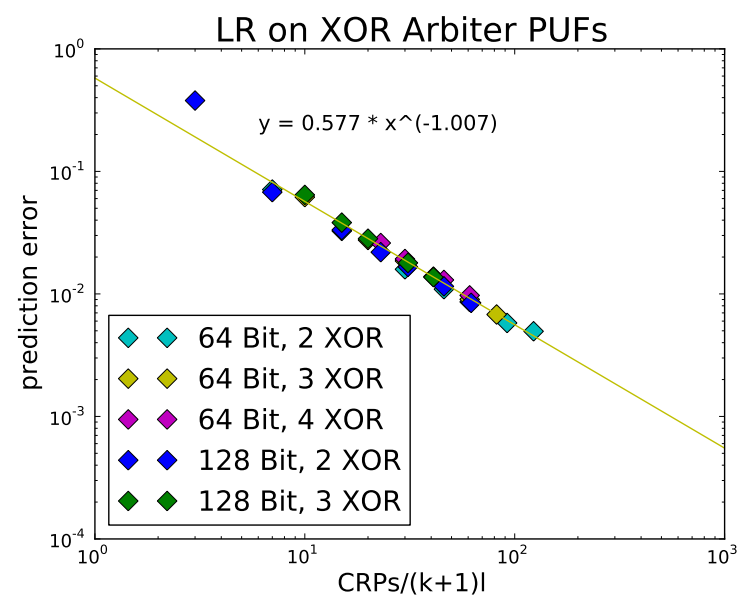

Figure 4: Double logarithmic plot of misclassification rate $\epsilon$ on the ratio of training CRPs $N_{C R P}$ and problem size $\operatorname{dim}(\Phi)=(k+1) \cdot l$. We used HW $\mathbf{~}$.

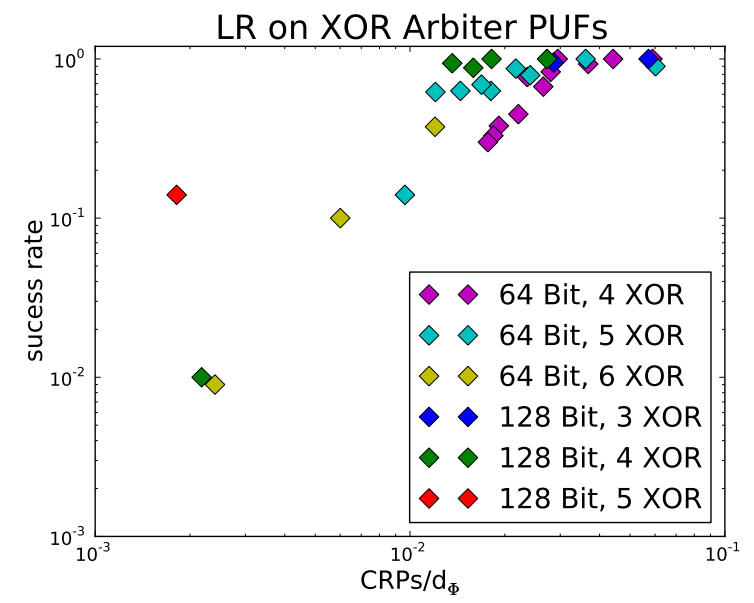

Figure 5: Average rate of success of the LR algorithm plotted in dependence of the ratio $d_{\Phi}$ (see Eqn. (13)) to $N_{C R P}$. We used HW $\mathbf{}$.

number of independent dimensions of $\vec{\Phi}_{X O R}=\bigotimes_{i=1}^{l} \vec{\Phi}_{i}=$ $\bigotimes_{i=1}^{l}\left(\Phi_{i}^{1} \ldots, \Phi_{i}^{k}, 1\right)^{T}$.

As the tensor product of several vectors consists of all possible products between their vector components, the independent dimensions are given by the number of different products of the form $\Phi_{1}^{i_{1}} \cdot \Phi_{2}^{i_{2}} \cdot \ldots \Phi_{l}^{i_{l}}$ for $i_{1}, i_{2}, \ldots, i_{l} \in$ $\{1,2, \ldots, k+1\}$ (where we say that $\Phi_{i}^{k+1}=1$ for all $i=$ $1, \ldots, l)$. For XOR Arb-PUFs, we furthermore know that the same challenge is applied to all $l$ internal Arbiter PUFs, which tells us that $\Phi_{j}^{i}=\Phi_{j^{\prime}}^{i}=\Phi^{i}$ for all $j, j^{\prime} \in\{1, \ldots, l\}$ and $i \in\{1, \ldots, k+1\}$. Since a repetition of one component does not affect the product regardless of its value (recall that $\Phi^{r} \cdot \Phi^{r}= \pm 1 \cdot \pm 1=1$ ), the number of the above products can be obtained by counting the unrepeated components. The number of different products of the above form is therefore given as the number of $l$-tuples without repetition, plus the number of $(l-2)$-tuples without repetition (corresponding to all $l$-tuples with 1 repetition), plus the number of $(l-4)$ tuples without repetition (corresponding to all $l$-tuples with 2 repetitions), etc.

Writing this down more formally, $d_{\Phi}$ is given by

$$
\begin{aligned}
& d_{\Phi}=\left(\begin{array}{c}
k+1 \\
l
\end{array}\right)+\left(\begin{array}{c}
k+1 \\
l-2
\end{array}\right)+\left(\begin{array}{c}
k+1 \\
l-4
\end{array}\right)+\ldots \\
& \geqq l l \\
& \stackrel{(k+1)^{l}}{l !} .
\end{aligned}
$$

The approximation applies when $k$ is considerably larger than $l$, which holds for the considered PUFs for stability reasons. Following [20], this seems to lead to an expected number of restarts $N_{\text {trial }}$ to obtain a valid decision boundary on the training set (that is, a parameter set $\vec{w}$ that separates the training set), of

$$
N_{\text {trial }}=O\left(\frac{d_{\Phi}}{N_{C R P}}\right)=O\left(\frac{(k+1)^{l}}{N_{C R P} \cdot l !}\right) .
$$

Furthermore, each trial has the complexity

$$
T_{\text {trial }}=O\left((k+1) \cdot l \cdot N_{C R P}\right) .
$$




\begin{tabular}{|c|c|c|c|c|}
\hline $\begin{array}{c}\text { No. of } \\
\text { Stages }\end{array}$ & $\begin{array}{c}\text { Pred. } \\
\text { Rate }\end{array}$ & $\begin{array}{c}\text { No. of } \\
\text { XORs }\end{array}$ & CRPs & $\begin{array}{c}\text { Training } \\
\text { Time }\end{array}$ \\
\hline \multirow{3}{*}{64} & & 3 & 6,000 & $8.9 \mathrm{sec}$ \\
& $99 \%$ & 4 & 12,000 & $1: 28 \mathrm{hrs}$ \\
& & 5 & 300,000 & $13: 06 \mathrm{hrs}$ \\
\hline \multirow{3}{*}{128} & \multirow{2}{*}{$99 \%$} & 3 & 15,000 & $40 \mathrm{sec}$ \\
& & 5 & 500,000 & $59: 42 \mathrm{~min}$ \\
& & 5 & $10^{6}$ & 267 days \\
\hline
\end{tabular}

Table 5: LR on Lightweight PUFs. Prediction rate refers to single output bits. Training times were averaged over different PUF instances. HW $\star$.

\section{LIGHTWEIGHT SECURE PUFS}

\subsection{Machine Learning Results}

In order to test the influence of the specific input mapping of the Lightweight PUF on its machine-learnability (see Sec. 2.3), we examined architectures with the following parameters: variable $l, m=1, x=l$, and arbitrary $s$. We focused on LR right from the start, since this method was best in class for XOR Arb-PUFs, and obtained the results shown in Table 5. The specific design of the Lightweight PUF improves its ML resilience by a notable quantitative factor, especially with respect to the training times and CRPs. The given training times and prediction rates relate to single output bits of the Lightweight PUF.

\subsection{Scalability}

Some theoretical consideration [20] shows the underlying ML problem for the Lightweight PUF and the XOR Arb PUF are similar with respect to the required CRPs, but differ quantitatively in the resulting runtimes. The asymptotic formula on $N_{C R P}$ given for the XOR Arb PUF (Eqn. 12) analogously also holds for the Lightweight PUF. But due to the influence of the special challenge mapping of the Lightweight PUF, the number $N_{\text {trial }}$ has a growth rate that is different from Eqn. 14. It seems to lie between $\left.O\left(\frac{(k+1)^{l}}{N_{C R P} \cdot l !}\right)\right)$ and the related expression $O\left(\frac{(k+1)^{l}}{N_{C R P}}\right)[20]$. While these two formulas differ by factor of $l$ !, we note that in our case $k \gg l$, and that $l$ is comparatively small for stability reasons. Again, all these considerations on $N_{C R P}$ and $N_{\text {Trial }}$ hold for the prediction of single output bits of the Lightweight PUF.

These points were at least qualitatively confirmed by our scalability experiments. We observed in agreement with the above discussion that with the same ratio $C R P s / d_{\Phi}$ the LR algorithm will have a longer runtime for the Lightweight PUF than for the XOR Arb-PUF. For example, while with a training set size of 12,000 for the 64-bit 4-XOR Arb-PUF on average about 5 trials were sufficient, for the corresponding Lightweight PUF 100 trials were necessary. The specific challenge architecture of the Lightweight PUF hence noticeably complicates the life of an attacker in practice.

\section{FEED FORWARD ARBITER PUFS}

\subsection{Machine Learning Results}

We experimented with SVMs and LR on FF Arb-PUFs, using different models and input representations, but could

\begin{tabular}{|c|c|c|c|c|}
\hline $\begin{array}{c}\text { No. of } \\
\text { Stages }\end{array}$ & $\begin{array}{c}\text { FF- } \\
\text { loops }\end{array}$ & $\begin{array}{c}\text { Pred. Rate } \\
\text { Best Run }\end{array}$ & CRPs & $\begin{array}{c}\text { Training } \\
\text { Time }\end{array}$ \\
\hline & 6 & $97.72 \%$ & 50,000 & $07: 51 \mathrm{~min}$ \\
64 & 7 & $99.38 \%$ & 50,000 & $47: 07 \mathrm{~min}$ \\
& 8 & $99.50 \%$ & 50,000 & $47: 07 \mathrm{~min}$ \\
& 9 & $98.86 \%$ & 50,000 & $47: 07 \mathrm{~min}$ \\
& 10 & $97.86 \%$ & 50,000 & $47: 07 \mathrm{~min}$ \\
\hline \multirow{6}{*}{128} & 6 & $99.11 \%$ & 50,000 & $3: 15 \mathrm{hrs}$ \\
& 7 & $97.43 \%$ & 50,000 & $3: 15 \mathrm{hrs}$ \\
& 8 & $98.97 \%$ & 50,000 & $3: 15 \mathrm{hrs}$ \\
& 9 & $98.78 \%$ & 50,000 & $3: 15 \mathrm{hrs}$ \\
& 10 & $97.31 \%$ & 50,000 & $3: 15 \mathrm{hrs}$ \\
\hline
\end{tabular}

Table 6: ES on Feed-Forward Arbiter PUFs. Prediction rates are for the best of a total of 40 trials on a single, randomly chosen PUF instance. Training times are for a single trial. We applied Lazy Evaluation with 2,000 CRPs. We used HW $\square$.

only break special cases with small numbers of non-overlapping FF loops, such as $l=1,2$. This is in agreement with earlier results reported in [19].

The application of ES finally allowed us to tackle much more complex FF-architectures with up to 8 FF-loops. All loops have equal length, and are distributed regularly over the PUF, with overlapping start- and endpoints of successive loops, as described in Section 2.3. Table 6 shows the results we obtained. The given prediction rates are the best of 40 trials on one randomly chosen PUF-instance of the respective length. The given CRP numbers are the sum of the training set and the test set employed by the attacker; a fraction of $5 / 6$ was used as the training set, $1 / 6$ as the test set (see Section 2.4). We note for comparison that in-silicon implementations of 64-bit FF Arb-PUFs with 7 FF-loops are known to have an environmental stability of $90.16 \%$ [17].

\subsection{Results on Error-Inflicted CRPs}

For the same reasons as in Section 4.2, we evaluated the performance on error-inflicted CRPs with respect to ES and FF Arb PUFs. The results are shown in Table 7 and Fig. 6. ES possesses an extremely high tolerance against the inflicted errors; its performance is hardly changed at all.

\subsection{Scalability}

We started by empirically investigating the CRP growth as a function of the number of challenge bits, examining archi-

\begin{tabular}{|c|c|c|c|c|c|}
\hline CRPs & & \multicolumn{4}{|c|}{ Percentage of error-inflicted CRPs } \\
\cline { 3 - 6 }$\left(\times 10^{3}\right)$ & & $0 \%$ & $2 \%$ & $5 \%$ & $10 \%$ \\
\hline \multirow{3}{*}{50} & Best Pred. & $98.29 \%$ & $97.78 \%$ & $98.33 \%$ & $97.68 \%$ \\
& Aver. Pred. & $89.94 \%$ & $88.75 \%$ & $89.09 \%$ & $87.91 \%$ \\
& Succ. Trials & $42.5 \%$ & $37.5 \%$ & $35.0 \%$ & $32.5 \%$ \\
\hline
\end{tabular}

Table 7: ES on 64-bit, 6 FF Arb PUFs with different levels of error in the training set. We show the best and average prediction rates from over 40 independent trials on a single, randomly chosen PUF instance, and the percentage of successful trials that converged to $90 \%$ or better. We used HW $\square$. 


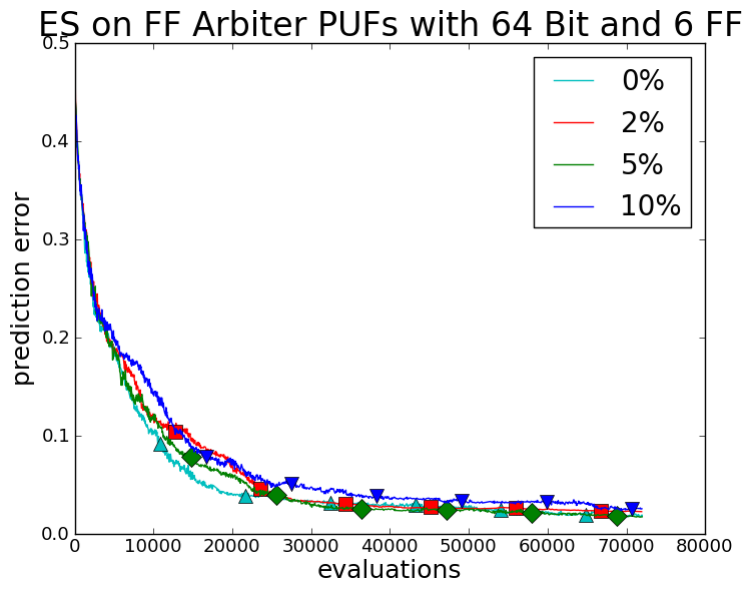

Figure 6: Graphical illustration of the tolerance of ES to errors. We show the best result of 40 independent trials on one randomly chosen PUF instance for varying error levels in the training set. The results hardly differ. We used HW $\square$.

tectures of varying bitlength that all have 6 FF-loops. The loops are distributed as described in Section 2.3. The corresponding results are shown in Figure 7. Every data point corresponds to the averaged prediction error of 10 trials on the same, random PUF-instance.

Secondly, we investigated the CRP requirements as a function of a growing number of FF-loops, examining architectures with 64 bits. The corresponding results are depicted in Figure 8. Again, each data point shows the averaged prediction error of 10 trials on the same, random PUF instance.

In contrast to the Sections 4.3 and 5.2, it is now much more difficult to derive reliable scalability formulas from this data. The reasons are threefold. First, the structure of ES provides less theoretical footing for formal derivations. Second, the random nature of ES produces a very large variance in the

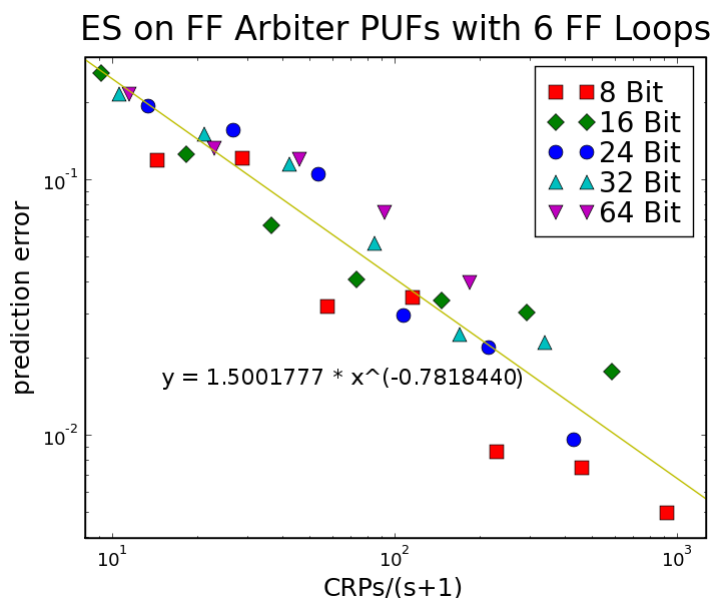

Figure 7: Results of 10 trials per data point with ES for different lengths of FF Arbiter PUFs and the hyperbola fit. HW $\mathrm{H}$.

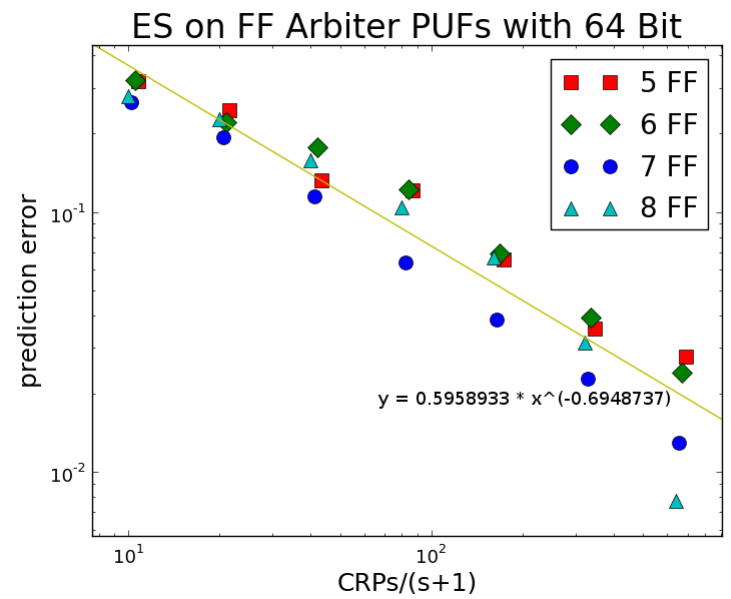

Figure 8: Results of 10 trials per data point with ES for different numbers of FF-loops and the hyperbola fit. HW $\square$.

data points, making also clean empirical derivations more difficult. Third, we observed an interesting effect when comparing the performance of ES vs. SVM/LR on the Arb PUF: While the supervised ML methods SVM and LR showed a linear relationship between the prediction error $\epsilon$ and the required CRPs even for very small $\epsilon$, ES proved more CRP hungry in these extreme regions for $\epsilon$, clearly showing a superlinear growth. The same effect can be expected for FF architectures, meaning that one consistent formula for extreme values of $\epsilon$ may be difficult to obtain.

It still seems somewhat suggestive from the data points in Figures. 7 and 8 to conclude that the growth in CRPs is about linear, and that the computation time grows polynomially. For the reasons given above, however, we would like to remain conservative, and present the upcoming empirical formulas only in the status of a conjecture.

The data gathered in our experiments is best explained by assuming a qualitative relation of the form

$$
N_{C R P}=O\left(s / \epsilon^{c}\right)
$$

for some constant $0<c<1$, where $s$ is the number of stages in the PUF. Concrete estimation from our data points leads to an approximate formula of the form

$$
N_{C R P} \approx 9 \cdot \frac{s+1}{\epsilon^{3 / 4}} .
$$

The computation time required by ES is determined by the following factors: (i) The computation of the vector product $\vec{w}^{T} \vec{\Phi}$, which grows linearly with $s$. (ii) The evolution applied to this product, which is negligible compared to the other steps. (iii) The number of iterations or "generations" in ES until a small misclassification rate is achieved. We conjecture that this grows linearly with the number of multiplexers $s$. (iv) The number of CRPs that are used to evaluate the individuals per iteration. If Eqn. 17 is valid, then $N_{C R P}$ is on the order of $O\left(s / \epsilon^{c}\right)$.

Assuming the correctness of the conjectures made in this derivation, this would lead to a polynomial growth of the 


\begin{tabular}{|c|c|c|c|c|c|}
\hline Method & $\begin{array}{c}\text { No. of } \\
\text { Oscill. }\end{array}$ & \multicolumn{2}{|c|}{$\begin{array}{c}\text { Pred. Rate } \\
\text { average }\end{array}$} & \multicolumn{2}{c|}{ CRPs } \\
\hline \multirow{3}{*}{ QS } & 256 & $99 \%$ & $99.9 \%$ & 14,060 & 28,891 \\
& 512 & $99 \%$ & $99.9 \%$ & 36,062 & 103,986 \\
& 1024 & $99 \%$ & $99.9 \%$ & 83,941 & 345,834 \\
\hline
\end{tabular}

Table 8: Quick Sort applied to the Ring Oscillator PUF. The given CRPs are averaged over 40 trials. We used HW $\square$.

computation time in terms of the relevant parameters $k, l$ and $s$. It could then be conjectured that the number of basic computational operations $N_{B O P}$ obeys

$$
N_{B O P}=O\left(s^{3} / \epsilon^{c}\right)
$$

for some constant $0<c<1$.

\section{RING OSCILLATOR PUFS}

\subsection{Possible Attacks}

There are several strategies to attack a RO-PUF. The most straightforward attempt is a simple read out of all CRPs. This is easy, since there are just $k(k-1) / 2=O\left(k^{2}\right)$ CRPs of interest, given $k$ ring oscillators.

If Eve is able to choose the CRPs adaptively, she can employ a standard sorting algorithm to sort the RO-PUF's frequencies $\left(f_{1}, \ldots, f_{k}\right)$ in ascending order. This strategy subsequently allows her to predict all outputs with $100 \%$ correctness, without knowing the exact frequencies $f_{i}$ themselves. The time and CRP complexities of the respective sorting algorithms are well known [27]; for example, there are several algorithms with average- and even worst-case CRP complexity of $N_{C R P}=O(k \cdot \log k)$. Their running times are also low-degree polynomial.

The most interesting case for our investigations is when Eve cannot adaptively choose the CRPs she obtains, but still wants to achieve optimal prediction rates. This case occurs in practice whenever Eve obtains her CRPs from protocol eavesdropping, for example. We carried out experiments for this case, in which we applied Quick Sort (QS) to randomly drawn CRPs. The results are shown in Table 8. The estimated required number of CRPs is given by

$$
N_{C R P} \approx \frac{k(k-1)(1-2 \epsilon)}{2+\epsilon(k-1)}
$$

and the training times are low-degree polynomial. Eqn. 19 quantifies limited-count authentication capabilities of ROPUFs.

\section{SUMMARY AND DISCUSSION}

Summary. We investigated the resilience of currently published electrical Strong PUFs against modeling attacks. To that end, we applied various machine learning techniques to challenge-response data generated pseudo-randomly via an additive delay model. Some of our main results are summarized in Table 9.

We found that all examined Strong PUF candidates under a given size could be machine learned with success rates above their in-silicon stability. The attacks require a number of CRPs that grows only linearly or log-linearly in the internal parameters of the PUFs, such as their number of stages, XORs, feed-forward loops or ring oscillators. Apart from XOR Arbiter PUFs and Lightweight PUFs (whose training times grew quasi-exponentially in their number of XORs for large bitlengths $k$ and small to medium number of XORs $l$ ), the training times of the applied machine learning algorithms are low-degree polynomial, too.

While we have presented results only on pseudo-random CRP data generated in the additive delay model, experiments with silicon implementations [17] [28] have shown that the additive delay model achieves very high accuracy. We also showed that the stability of our results against random errors in the CRP data is high. Our approach is hence robust against some inaccuracies in the model and against measurement noise. In our opinion, it will transfer to the case where CRP data is collected from silicon PUF chips.

Our results prohibit the use of the broken architectures as Strong PUFs or in Strong-PUF based protocols. Under the assumption that digital signals can be probed, they also affect the applicability of the cryptanalyzed PUFs as building blocks in Controlled PUFs and Weak PUFs.

Discussion. Two straightforward, but biased interpretations of our results would be the following: (i) All Strong PUFs are insecure. (ii) The long-term security of electrical Strong PUFs can be restored trivially, for example by increasing the PUF's size. Both views are simplistic, and the truth is more involved.

Starting with (i), our current attacks are indeed sufficient to break most implemented PUFs. But there are several ways how PUF designers can fight back in future implementations. First, increasing the bitlength $k$ in an XOR Arbiter PUF or Lightweight Secure PUF with $l$ XORs increases the effort of the presented attacks methods as a polynomial function of $k$ with exponent $l$ (in approximation for large $k$ and small or medium $l$ ). At the same time, it does not worsen the PUF's stability [28]. For now, one could therefore disable attacks through choosing a strongly increased value of $k$ and a value of $l$ that corresponds to the stability limit of such a construction. For example, an XOR Arbiter PUF with 8 XORs and bitlength of 512 is implementable by standard fabrication processes [28], but is currently beyond the reach of our attacks. Similar considerations hold for Lightweight PUFs of these sizes. Secondly, new design elements may raise the attacker's complexity further, for example adding nonlinearity (such as AND and OR gates that correspond to MAX and MIN operators [17]). Combinations of Feed-

\begin{tabular}{|c|c|c|c|c|c|c|}
\hline $\begin{array}{c}\text { PUF } \\
\text { Type }\end{array}$ & $\begin{array}{c}\text { XORs/ } \\
\text { Loops }\end{array}$ & $\begin{array}{c}\text { ML } \\
\text { Met. }\end{array}$ & $\begin{array}{c}\text { No.of } \\
\text { Stag. }\end{array}$ & $\begin{array}{c}\text { Pred. } \\
\text { Rate }\end{array}$ & $\begin{array}{c}\text { CRPs } \\
\left(\times 10^{3}\right)\end{array}$ & $\begin{array}{c}\text { Train. } \\
\text { Time }\end{array}$ \\
\hline Arb & - & LR & 128 & $99.9 \%$ & 39.2 & 2.10 sec \\
\hline XOR & 5 & LR & 128 & $99.0 \%$ & 500 & $16: 36 \mathrm{hrs}$ \\
\hline Light & 5 & LR & 128 & $99.0 \%$ & 1000 & 267 days \\
\hline FF & 8 & ES & 128 & $99.0 \%$ & 50 & $3: 15$ hrs \\
\hline
\end{tabular}

Table 9: Some of our main results. 
Forward and XOR architectures could be hard to machine learn too, partly because they seem susceptible only to different and mutually-exclusive ML techniques.

Moving away from delay-based PUFs, the exploitation of the dynamic characteristics of current and voltage seems promising, for example in analog circuits [29]. Also special PUFs with a very high information content (so-called SHIC PUFs $[30,31,32])$ could be an option, but only in such applications where their slow read-out speed and their comparatively large area consumption are no too strong drawbacks. Their promise is that they are naturally immune against modeling attacks, since all of their CRPs are informationtheoretically independent. Finally, optical Strong PUFs, for example systems based on light scattering and interference phenomena [1], show strong potential in creating high inputoutput complexity.

Regarding view (ii), PUFs are different from classical cryptoschemes like RSA in the sense that increasing their size often likewise decreases their input-output stability. For example, raising the number of XORs in an XOR Arbiter PUF has an exponentially strong effect both on the attacker's complexity and on the instability of the PUF. We are yet unable to find parameters that increase the attacker's effort exponentially while affecting the PUF's stability merely polynomially. Nevertheless, one practically viable possibility is to increase the bitlength of XOR Arbiter PUFs, as discussed above. Future work will have to show whether the described large polynomial growth can persist in the long term, or whether its high degree can be diminished by further analysis.

Future Work. The upcoming years will presumably witness an intense competition between codemakers and codebreakers in the area of Strong PUFs. Similar to the design of classical cryptoprimitives, for example stream ciphers, this process can be expected to converge at some point to solutions that are resilient against the known attacks.

For PUF designers, it may be interesting to investigate some of the concepts that we mentioned above. For PUF breakers, a worthwhile starting point is to improve the attacks presented in this paper through optimized implementations and new ML methods. Another, qualitatively new path is to combine modeling attacks with information obtained from direct physical PUF measurements or from side channels. For example, applying the same challenge multiple times gives an indication of the noise level of a response bit. It enables conclusions about the absolute value of the final runtime difference in the PUF. Such side channel information can conceivably improve the success and convergence rates of ML methods, though we have not exploited this in this paper.

\section{Acknowledgements}

This work was partly supported by the Physical Cryptography Project of the Technische Universität München.

\section{REFERENCES}

[1] R. Pappu, B. Recht, J. Taylor, and N. Gershenfeld. Physical one-way functions. Science, 297(5589):2026, 2002.

[2] B. Gassend, D. Clarke, M. Van Dijk, and S. Devadas. Silicon physical random functions. In Proceedings of the 9th ACM Conference on Computer and Communications Security, page 160. ACM, 2002.

[3] Blaise Gassend, Dwaine Clarke, Marten van Dijk, and Srinivas Devadas. Controlled physical random functions. In Proceedings of 18th Annual Computer Security Applications Conference, Silver Spring, MD, December 2002.

[4] J. Guajardo, S. Kumar, G.J. Schrijen, and P. Tuyls. FPGA intrinsic PUFs and their use for IP protection. Cryptographic Hardware and Embedded Systems-CHES 2007, pages 63-80, 2007.

[5] B.L.P. Gassend. Physical random functions. Msc thesis, MIT, 2003.

[6] R. Pappu. Physical One-Way Functions. Phd thesis, MIT, 2001.

[7] P. Tuyls and B. Skoric. Strong Authentication with PUFs. In: Security, Privacy and Trust in Modern Data Management, M. Petkovic, W. Jonker (Eds.), Springer, 2007.

[8] Ulrich Rührmair. Oblivious transfer based on physical unclonable functions (extended abstract). In Alessandro Acquisti, Sean W. Smith, and Ahmad-Reza Sadeghi, editors, TRUST, volume 6101 of Lecture Notes in Computer Science, pages 430-440. Springer, 2010.

[9] G.E. Suh and S. Devadas. Physical unclonable functions for device authentication and secret key generation. Proceedings of the 44th annual Design Automation Conference, page 14, 2007.

[10] M. Majzoobi, F. Koushanfar, and M. Potkonjak. Lightweight secure pufs. In Proceedings of the 2008 IEEE/ACM International Conference on Computer-Aided Design, pages 670-673. IEEE Press, 2008.

[11] B. Gassend, D. Lim, D. Clarke, M. Van Dijk, and S. Devadas. Identification and authentication of integrated circuits. Concurrency and Computation: Practice \&S Experience, 16(11):1077-1098, 2004.

[12] J.W. Lee, D. Lim, B. Gassend, G.E. Suh, M. Van Dijk, and S. Devadas. A technique to build a secret key in integrated circuits for identification and authentication applications. In Proceedings of the IEEE VLSI Circuits Symposium, pages 176-179, 2004.

[13] D. Lim, J.W. Lee, B. Gassend, G.E. Suh, M. Van Dijk, and S. Devadas. Extracting secret keys from integrated circuits. IEEE Transactions on Very Large Scale Integration Systems, 13(10):1200, 2005.

[14] Daniel E. Holcomb, Wayne P. Burleson, and Kevin Fu. Initial sram state as a fingerprint and source of true random numbers for rfid tags. In In Proceedings of the Conference on RFID Security, 2007.

[15] S.S. Kumar, J. Guajardo, R. Maes, G.J. Schrijen, and P. Tuyls. Extended abstract: The butterfly PUF protecting IP on every FPGA. In IEEE International Workshop on Hardware-Oriented Security and Trust, 2008. HOST 2008, pages 67-70, 2008. 
[16] P. Tuyls, G.J. Schrijen, B. Škorić, J. van Geloven, N. Verhaegh, and R. Wolters. Read-proof hardware from protective coatings. Cryptographic Hardware and Embedded Systems-CHES 2006, pages 369-383, 2006.

[17] Daihyun Lim. Extracting Secret Keys from Integrated Circuits. Msc thesis, MIT, 2004.

[18] Erdinç Öztürk, Ghaith Hammouri, and Berk Sunar. Towards robust low cost authentication for pervasive devices. In PerCom, pages 170-178. IEEE Computer Society, 2008.

[19] M. Majzoobi, F. Koushanfar, and M. Potkonjak. Testing techniques for hardware security. In Proceedings of the International Test Conference (ITC), pages 1-10, 2008.

[20] Jan Sölter. Cryptanalysis of Electrical PUFs via Machine Learning Algorithms. Msc thesis, Technische Universität München, 2009.

[21] C.M. Bishop et al. Pattern recognition and machine learning. Springer New York:, 2006.

[22] M. Riedmiller and H. Braun. A direct adaptive method for faster backpropagation learning: The RPROP algorithm. In Proceedings of the IEEE international conference on neural networks, volume 1993, pages 586-591. San Francisco: IEEE, 1993.

[23] http://www.pcp.in.tum.de/code/lr.zip, 2010.

[24] T. Bäck. Evolutionary algorithms in theory and practice: evolution strategies, evolutionary programming, genetic algorithms. Oxford University Press, USA, 1996.

[25] H.P.P. Schwefel. Evolution and Optimum Seeking: The Sixth Generation. John Wiley \& Sons, Inc. New York, NY, USA, 1993.

[26] T. Schaul, J. Bayer, D. Wierstra, Y. Sun, M. Felder, F. Sehnke, T. Rückstieß, and J. Schmidhuber. PyBrain. Journal of Machine Learning Research, 1:999-1000, 2010.

[27] C.H. Papadimitriou. Computational complexity. John Wiley and Sons Ltd., 2003.

[28] S. Devadas. Physical unclonable functions and secure processors. In Workshop on Cryptographic Hardware and Embedded Systems (CHES 2009), September 2009.

[29] G. Csaba, X. Ju, Z. Ma, Q. Chen, W. Porod, J. Schmidhuber, U. Schlichtmann, P. Lugli, and U. Rührmair. Application of mismatched cellular nonlinear networks for physical cryptography. In 12th IEEE CNNA - International Workshop on Cellular Nanoscale Networks and their Applications. Berkeley, CA, USA, February 3 - 52010.

[30] U. Rührmair, C. Jaeger, M. Bator, M. Stutzmann, P. Lugli, and G. Csaba. Applications of high-capacity crossbar memories in cryptography. To appear in IEEE Transactions on Nanotechnology, 2010.

[31] U. Rührmair, C. Jaeger, C. Hilgers, M. Algasinger, G. Csaba, and M. Stutzmann. Security applications of diodes with unique current-voltage characteristics. In Lecture Notes in Computer Science, volume 6052, Tenerife (Spain), January 25 - 28 2010. 14th International Conference on Financial Cryptography and Data Security, Springer.

[32] C. Jaeger, M. Algasinger, U. Rührmair, G. Csaba, and M. Stutzmann. Random p-n-junctions for physical cryptography. Applied Physics Letters, 96(172103), 2010 . 